\title{
Research Progress on Trypanosomiasis in China
}

\author{
Sihong Shen, Jianfa Liu* \\ Ningbo University School of Medicine, Ningbo, China \\ Email: ^liujianfa@nbu.edu.cn
}

How to cite this paper: Shen, S.H. and Liu, J.F. (2017) Research Progress on Trypanosomiasis in China. Open Access Library Journal, 4: e4118.

https://doi.org/10.4236/oalib.1104118

Received: November 3, 2017

Accepted: November 17, 2017

Published: November 20, 2017

Copyright ( $\odot 2017$ by authors and Open Access Library Inc.

This work is licensed under the Creative Commons Attribution International License (CC BY 4.0).

http://creativecommons.org/licenses/by/4.0/

\begin{abstract}
In this paper, the research on biological characteristics, the immunological antigens of trypanosomes, the molecular biological researches on the mechanism of trypanosomiasis, and the study of chemotherapy on trypanosomiasis have been summarized. The prospects of trypanosome and trypanosomiasis have been provided.
\end{abstract}

\section{Subject Areas}

Infectious Diseases, Public Health

\section{Keywords}

Trypanosomiasis, Immunology, Molecular Biology, Chemotherapy

\section{Introduction}

Trypanosome is a class of protozoa with flagella, belonging to the movement matrix, such as trypanosome and trypanosomiidae. Trypanosomiasis is common in the blood and cells of mammals, birds, fish and amphibians. It is mainly transmitted through various blood-sucking insects [1]. So far, there are two types of trypanosomes, one of which is the development of salivary trypanosoma in the front of the digestive tract, which is usually pathogenic. The other is the faecal parasite, which develops at the back of the alimentary canal, in which all except the dead trypanosomiasis [2]. Trypanosomiasis has not been developed successfully in the host body by continuously changing surface antigens to avoid host immunity. Control of Trypanosomiasis can only rely on drugs. Due to the frequent use of a resistant mechanism of the same class of drugs, in order to prevent the formation of cone insect resistance, the insect body to the drug re- 
sistance could use biochemistry, molecular biology and gene engineering technology research trypanosome resistance mechanism. Although these studies has obtained certain achievements, there are still many details are not clear, so the resistance mechanism of trypan has much difficulty.

\section{The Morphological Study of Trypanosomes}

Most trypanosomes tapering at both ends, and has developed the volatility of the membrane and a long-standing free flagella, of course, there are the kinds of short-term exist flagella, and some species have an obvious ovoid nuclei and a vacuolated cytoplasm [3].

\subsection{Surface Membrane}

The trypanosoma is a protozoan, composed of individual cells. The membrane is a layer of protoplasm wrapped in the surface of the cone, and the outer layer of the membrane is covered by a coat membrane that protects the body from the immune function of the host.

\subsection{Surface Variation Glycoprotein}

Trypan chronic infection in the host usually can last for years, changing its rely on surface cover coat membrane antigen, so as to achieve the aim of avoiding host immune function, this kind of antigen variation phenomenon is an important biological characteristics, trypanosome coat layer covering the surface of the film is actually surface glycoprotein.

\subsection{The Nucleus}

Trypanosoma usually has only one nucleus, in the cytoplasm, usually round or oval. Nuclear membrane can be divided into two layers of nuclear membrane and nuclear membrane, nuclear membrane on the nuclear pore, as small molecules and ions free through the channel, there are many free ribosomes under nuclear membrane, the cell nucleus contains a central nucleoli. After the nucleus of the nucleus was stained by Jim sa, it was observed in dark purple.

\subsection{The Moving Substrate}

The moving matrix is a special structure in the mitochondria of trypanosome, also known as the active nucleus, usually short, round, or elliptical. The moving matrix contains self-replicating DNA, which is surrounded by large circular DNA and small circular DNA molecules. Due to the dynamic matrix is located in the mitochondria, but store the rich can staining DNA structure, dynamic matrix is peculiar to the parasite structure, dynamic matrix, however, its function has not been elucidated in trypanosome body.

\subsection{The Flagellum}

The flagellum protrudes from the posterior extremity of the body of the body in 
the back end of the body, extending from the back end of the body to the front end of the body. Trypanosome flagella outsourcing one layer of coat film, outer ring for 9 group of duplex microtubules, package within a pair of central microtubules, is a typical structure of $9+2$ microtubules, have a lattice of balance beam at the same time, between the tubes and balance beam inside also has a lot of empty area. The flagella can be continuously contracted through the thread structure in the central microtube, which can promote the movement of trypanosome.

\subsection{Undulating Membrane}

The undulating membrane is a unique structure of trypanosome, and most of them have obvious undulating membrane structures, usually wavy or finned. Vickerman observes via ultrastructure that the wave membrane is derived from the membrane of the membrane, rather than a part of the flagellum [4].

\section{Immunological Research}

Since trypanosome has strong surface antigenic ability to mutate and evade the host immune attack, there is no ideal vaccine for practical application. Tabular mutated glycoprotein (VSG) is a recognized strong immunogen, which can stimulate the host to produce cell and humoral immune response, and the elimination of trypanosomiasis is mainly dependent on VSG antibody mediated. The immunological prevention of trypanosomiasis has been the focus of scholars in China.

Studies showed ShTat1.1 VSG + ShTat1.2 VSG compound antigen immunizing mice can avoid the interference of trypanosome antigen variation to obtain good immune protection effect, and with ShTat1.1 VSG immune mice can only get part of the immune protection, cannot overcome the interference of trypanosome antigen mutation, immune rat on the top by trypanosome ShTat1.1 VSG stimulate immunity can only kill most take corresponding variant trypanosoma, in a short period of time had failed to kill a few variation for trypan ShTat1.2 trypan, evade the host immune, and multiply, and kill the rat [5].

There are also studies showing that trypanosomal multiple miRNA families can regulate the variable surface glycoprotein antigen mRNA of trypanosomiasis [6]. TongXinXin found that IL 27 can direct inhibition of CD4 + T cell proliferation and Th2 cytokines, that IL - 27 - WSX - 1 type of mucosal sites II has inhibitory effect on the inherent immunity and adaptive immunity, and WSX - 1 for t. cruzi parasites Th1 response during the period of infection is very important to start [7]. There are also some scholars who use the surface antigen specificity of trypanosomiasis to develop monoclonal antibody against the VSG antigens of the ichis, and develop the immune-colloidal gold test paper with the positive coincidence rate of $88.2 \%$. The rapid, sensitive and specific detection of the water buffalo's trypanosomiasis is an effective method for the detection and precontrol of the detection and precontrol of ichistrypanosomiasis of buffalo [8]. 


\section{Molecular Biology Research}

Trypanosoma is susceptible to drug resistance, and the resistance of trypanosomiasis has been documented in 1908. It is the first class of parasite to detect drug resistance [9]. In order to guide the proper use of drugs and explore prevent cone insect resistance and remove the existing drug resistance, many scholars of developed countries and developing countries, the cone insect resistance mechanism of biochemical research. The study mainly has three directions, the enzyme activity changes of drug resistant trypanosomiasis [10]; Adenosine transporters [11]; Genetic changes [12].

El-Sayed [13] in 400 cDNAs and random of genome DNA chip to display brinell trypanosome gene expression in the process of development, for the trypanosome infection, the discovery of new drug targets and the mechanism of host candidate vaccine development provides the basis. Subsequently, a number of researchers used gene chip technology to study the specific gene expression in different developmental periods, such as upper flagellum, and late cone flagellates. [14] [15] [16] [17] [18]. Study shows that the trypanosome mitochondria may play an important role in regulating its life cycle [19].

Researchers found brinelltrypan natural trans transcribed sequence in different life period expression differences and mitochondrial RNA editing the phenomenon to appear in the blood type, as well as explore the brinelltrypan differentiation mechanism provides a new theoretical basis [20].

\section{Research on Chemotherapy for Parasites}

Because of the antigenic mutation of trypanosome, a better vaccine cannot be developed. So far, the prevention and control of trypanosomiasis has mostly relied on drugs. Although some progresses have been made in molecular biology research on the mechanism of trypanosomiasis resistance, it is still very limited. To break the limitation of conifer resistance, drug resistance is also needed to design the drug.

Myocardial histopathological observation results showed that the injection growth hormone could reduce the load without flagella and reduce the inflammatory infiltration and tissue structure destruction. The results showed that the growth hormone could be used as an immune regulator to control the cone-worm replication, and could be used in combination with the present drug to reduce the harm [21].

The research results show that with trypan bright ammonia acyl tRNAsynthetase for target drug trypan filter system can effectively screen trypan resistant compound, selected compounds have certain resistance trypan specificity, and can be used to compound further optimized and measure its half inhibitory concentration. Filter to use this system to the of trypan has good inhibition, but less poisonous to the cells of the human a series of new compounds, thus trypan bright ammonia acyl tRNAsynthetase is likely to become the new targets for the development of effective drug trypan [22]. 


\section{Prospects}

Trypanosome parasite and trypanosomiasis is common in tropical and sub-tropical areas. In China, there are rarely endemic. The accident rate and mechanism of this disease should be investigated in the future.

\section{Financial Support}

Financially supported by Scientific and planning programme of Zhejiang province (2017C37113). Subject programme Foundation of Ningbo University (XKL14D2105).

\section{References}

[1] Wu, H. (2016) Taxonomic Study of Four Freshwater Fish Trypanosomes. Journal of Huazhong Agricultural University, 12, 16-20.

[2] Schwede, A., Macleod, O.J., MacGregor, et al. (2015) How Does the VSG Coat of Bloodstream Form African Trypanosomes Interact with External Proteins. PLOS Pathogens, 11, 79-83. https://doi.org/10.1371/journal.ppat.1005259

[3] Wang, B., Yu, D.J. and Wang, X.L. (2011) Research Advances of Hepatozoon and Trypanosoma of the Amphibians. China Animal Health, 13, 20-25.

[4] Vickerman, K. (1969) On the Surface Coat and Flagellar Adhesion in Trypanosomes. Journal of Cell Science, 5, 163-193.

[5] Wang, Q., Shen, J., Zhou, Y.Z., et al. (2003) Regularity of Antigenic Variation in T. evansiin Mice and Experiments on Immune Protection According to Regularity of Antigenic Variation. Chinese Journal of Veterinary Science, 12, 160-163.

[6] Sun, Y. (2015) Research on Regulation of Trichinellaspriralis Excretory-Secretory Products by MicroRNAtsp-Novel-46. Journal of Jilin University, 18, 66-70.

[7] Tong, X.X. and Lv. F.L. (2014) Progress on IL-27 in Immunity to Important Protozoan Parasitic Infections. Chinese Journal of Parasitology and Parasitic Diseases, 32, 234-238.

[8] Meng, M., Cao, C., Chen, H.Z., et al. (2011) Preparation and Primary Application of Immunity Colloidal Gold Test Strip for Water Buffalo Trypanosomiasis. Chinese Journal of Animal and Veterinary Sciences, 42, 988-993.

[9] Coderre, A.R., Beverley, S.M. and Schimke, R.T. (1983) Overproduction of a Bifunctional Thymidylate. Synthetased-Dihydrofo-Late Reductase and DNA Amplification in Methotrexate-Rosistant. Lesihmaniatropica. Proceedings of the National Academy of Sciences, 80, 21-23. https://doi.org/10.1073/pnas.80.8.2132

[10] Boid, R. (1988) Isoenzyme Characterisation of 15 stocks of Try-Panosoma Evansi Isolated from Cames in the Sudar. Tropical Medicine and Parasitology, 39, 45-50.

[11] Carter, N.S. and Firlamb, A.H. (1993) Arsenical-Resistant Try-Panosomes Lack an Unusnal Adenosine Transporter. Nature, 361, 173-175.

https://doi.org/10.1038/361173a0

[12] Wilson, K., Berens, R., Sifri, C.D., et al. (1994) Amplification of the Inosinate Dehydrogenase Gene in Trypanosoma brucei gambiense Due to an Increase in Chromosome Copy Number. The Journal of Biological Chemistry, 269, 28979-28987.

[13] El-Sayed, N.M., Hegde, P., Quackenbush, J., et al. (2000) The African Trypanosome Genome. International Journal for Parasitology, 30, 329-345.

https://doi.org/10.1016/S0020-7519(00)00015-1 
[14] Diehl, S., Diehl, F., El-Sayed, N.M., et al. (2002) Analysis of Stage-Specific Gene Expression in the Bloodstream and the Procyclic form of Trypanosoma brucei Using a Genomic DNA-Microarray. Molecular and Biochemical Parasitology, 123, 115-123. https://doi.org/10.1016/S0166-6851(02)00138-X

[15] Jensen, B.C., Sivam, D., Kifer, C.T., et al. (2009) Widespread Variation in Transcript Abundance within and across Developmental Stages of Trypanosoma brucei. BMC Genomics, 10, 482. https://doi.org/10.1186/1471-2164-10-482

[16] Queiroz, R., Benz, C., Fellenberg, K., et al. (2009) Transcriptome Analysis of Differentiating Trypanosomes Reveals the Existence of Multiple Post-Transcriptional Regulons. BMC Genomics, 10, 495. https://doi.org/10.1186/1471-2164-10-495

[17] Simo, G., Herder, S., Cuny, G., et al. (2010) Identification of Subspecies Specific Genes Differentially Expressed in Procyclic Forms of Trypanosoma brucei Subspecies. Infection, Genetics and Evolution, 10, 229-237.

https://doi.org/10.1016/j.meegid.2009.11.003

[18] Veitch, N.J., Johnson, P.C., Trivedi, U., et al. (2010) Digital Gene Expression Analysis of Two Life Cycle Stages of the Human-Infective Parasite, Trypanosoma brucei gambiense Reveals Differentially Expressed Clusters of Co-Regulated Genes. BMC Genomics, 11, 124. https://doi.org/10.1186/1471-2164-11-124

[19] Zhang, X.B. (2011) Trypanosoma brucei MitoCarta and Its Expression Regulation. Nanjing University of Aeronautics and Astronautics, 22, 78-82.

[20] Wen, Y.Z. (2011) The Identification of Pseudogene-Derived siRNAs, NATs and the Function of MRB1 Complex Subunits in Trypanosoma rucei. Journal of Zhongshan University, 23, 12-20.

[21] Frare, E.O. and Zhang. Y.X. (2010) Hormone and Immunological Treatment of Cruze Trypanosomias. China Animal Husbandry \& Veterinary Medicine, 37, 213-216.

[22] Gao, G.W., Yao, Y., Ding, D.Z., Ye, L., Zhou, H.C. and Li. D.W. (2009) The Establishment of an Anti-Trypanosoma Drug Screening System with Leucyl-tRNA Synthetase as an Inhibition Target. China Biotechnology, 29, 13-17.

Submit or recommend next manuscript to OALib Journal and we will provide best service for you:

- Publication frequency: Monthly

- 9 subject areas of science, technology and medicine

- Fair and rigorous peer-review system

- Fast publication process

- Article promotion in various social networking sites (LinkedIn, Facebook, Twitter, etc.)

- Maximum dissemination of your research work

Submit Your Paper Online: Click Here to Submit

Or Contact service@oalib.com 